\title{
EQUATIONS OF MOTION THEORY FOR ELECTRON AFFINITIES
}

\section{Jack SiMONS}

Chemistry Department and Henry Eyring Center for Theoretical Chemistry, University of Utah, Salt Lake City, Utah 84112,U.S.A.; e-mail: simons@chemistry.utah.edu

One of this author's earliest independent achievements was inspired by his participation as a student at a Battelle-sponsored summer school held in Seattle, Washington at which Professor Paldus and many other pioneers of many-body methods in quantum chemistry were instructors. In particular, soon after arriving at the University of Utah in 1971, the author undertook a project aimed at utilizing many-body methods, specifically the equations of motion (EOM) point of view that McKoy and co-workers had applied to electronic excitations. His objective was to achieve computationally tractable working equations for an EOM-based method for directly computing molecular electron affinities. Much of his life-long involvement in studying anions derives from that work in 1971, which produced his first publication on the subject "Theory of Electron Affinities of Small Molecules" (Simons J., Smith W. D.: J. Chem. Phys. 1973, 58, 4899). For this reason, the author wishes to express his special debt and gratitude to Prof. J. Paldus and to wish him continued happiness and scientific success.

The ab initio calculation of molecular electron affinities (EA) and ionization potentials (IP) is a difficult task because the energy of interest is a very small fraction of the total electronic energy of the parent species. For example, EAs typically lie in the 0.01-10 eV range, but the total electronic energy of even a small molecule, radical, or ion is usually several orders of magnitude larger. Moreover, the EA or IP is an intensive quantity but the total energy is an extensive quantity, so the difficulty in evaluating EAs and IPs to within a fixed specified (e.g., $\pm 0.1 \mathrm{eV}$ ) accuracy becomes more and more difficult as the system's size and number of electrons grows. The situation becomes especially problematic when studying extended systems such as solids, polymers, or surfaces for which the EA or IP is an infinitesimal fraction of the total energy. EOM methods such as the author developed in the 1970s offer a route to calculating the intensive EAs and IPs directly as eigenvalues of a set of working equations. A history of the development of EOM theories as applied to EAs and IPs, their numerous practical implementations, and their relations to Greens function or propagator theories are given in this contribution. EOM methods based upon Møller-Plesset, multiconfiguration self-consistent field, and coupled-cluster reference wave functions are included in the discussion as is the application of EOM methods to metastable states of anions.

Keywords: Quantum chemistry; Koopmans' theorem; Coupled-cluster; EOM theory; MC-SCF; Metastable anion states. 
The vertical electron affinity (EA) of a molecule can be estimated by (approximately) solving the Schrödinger equation for the energy $E(0, N)$ of the $\mathrm{N}$-electron neutral molecule and the Schrödinger equation for the energy $E(K, N+1)$ of the $K$-th state of the $N+1$-electron anion and subtracting the two energies:

$$
E A=E(0, N)-E(K, N+1)
$$

The corresponding vertical ionization potential (IP) is given as

$$
I P=E(K, N-1)-E(0, N)
$$

Here, we use $\mathrm{K}$ to label the electronic state of the anion or cation that one wishes to study, and 0 to label the state of the neutral (usually but not necessarily the ground state) to which the electron is being attached or from which it is removed.

In using such an approach to obtaining the EA or IP, one is faced with a very difficult numerical challenge because $E(0, N), E(K, N-1)$, and $E(K, N+1)$ tend to be extremely large (negative) numbers, whereas EA and IP nearly always lie in the range $0-20 \mathrm{eV}$. For example, the EA of the ${ }^{4} \mathrm{~S}_{3 / 2}$ state of the

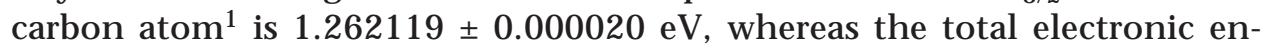
ergy of this state of $\mathrm{C}$ is $-1030.080 \mathrm{eV}$ (relative to a $\mathrm{C}^{6+}$ nucleus and six electrons infinitely distant and not moving that defines the zero of energy). Since the EA is ca. $0.1 \%$ of the total energy of $C$, one needs to compute the $\mathrm{C}$ and $\mathrm{C}^{-}$electronic energies to accuracies of $0.01 \%$ or better to calculate the EA to within $10 \%$.

However, the problem is even worse than this example suggests because $E(0, N), E(K, N-1)$, and $E(K, N+1)$ are extensive properties whereas $E A$ and IP are intensive quantities. For example, the EA of $\mathrm{C}_{2}$ in its $\mathrm{X}^{2} \Sigma_{\mathrm{g}}{ }^{+}$ground electronic state ${ }^{1}$ is $3.269 \pm 0.006 \mathrm{eV}$ near the equilibrium bond length $R_{e}$ but only $1.2621 \mathrm{eV}$ at $\mathrm{R} \rightarrow \infty$ (i.e., the same as the EA of a carbon atom). However, the total electronic energy of $\mathrm{C}_{2}$ is $-2060.160 \mathrm{eV}$ at $\mathrm{R} \rightarrow \infty$ and lower by ca. $3.6 \mathrm{eV}$ (the dissociation energy ${ }^{2}$ of $C_{2}$ ) at $R_{e}$, so again EA is a very small fraction of the total energies. For buckyball $C_{60}$, the EA is $2.666 \pm$ $0.001 \mathrm{eV}$, but the total electronic energy is sixty times $-1030.080 \mathrm{eV}$ minus the atomization energy (i.e., the energy change for $\mathrm{C}_{60} \rightarrow 60 \mathrm{C}$ ) of this compound. Clearly, the challenge of evaluating EA (or IP) to within even $50 \%$ 
becomes more and more difficult as the size (i.e., number of electrons) in the molecule grows, and it becomes impossible when the system of interest is an infinite solid, surface, or polymer. This same kind of difficulty (i.e., calculating an intensive quantity as the difference between two extensive energies) plagues the computation of EAs and of IPS, bond energies, and electronic excitation energies.

The problems discussed in the preceding paragraph do not disappear if one uses a computer with higher numerical precision (i.e., a longer word length) or algorithms that compute the one- and two-electron integrals to more significant figures. No matter how precise the integrals and how long the floating point word length (as long as they are finite), the evaluation of intensive properties such as IPS, EAs, and excitation energies as differences between pairs of extensive total electronic energies is doomed to fail.

Of course, much progress can be made in computing EAs and IPs as differences between anion and neutral or cation and neutral total energies ${ }^{3}$ be cause of large systematic cancellation in energy errors ${ }^{4}$. For example, the pair correlation energies of the two 1 s electron pairs in $C_{2}$ is quite large, but is very nearly the same as in $\mathrm{C}_{2}{ }^{-}$, so even a large per cent error made in computing these contributions to the total energy may not greatly affect the EA computed by subtracting $E(K, N+1)$ from $E(0, N)$. Some of the earliest high quality ab initio calculations of EAs were carried out using wave function techniques ${ }^{5}$ and calculating separate neutral and anion energies. Nevertheless, in the late 1960s and early 1970s, workers were motivated to develop methods that would allow intensive energy differences such as EAs, IPS and excitation energies $(\Delta \mathrm{E})$ "directly" rather than as differences in two very large numbers. This point of view is what led to the development of so-called equations of motion (EOM) methods as well as Greens function methods $^{6}$ and, more recently, response function approaches ${ }^{7}$. In all of these theories, one performs a derivation in which the two total energies (i.e., ion and neutral or ground and excited states) are subtracted analytically (rather than numerically) thereby achieving an analytical expression for the desired intensive energy difference. It is by thus dealing with equations that involve only intensive energies that one can overcome the problems detailed earlier.

Among the earliest practitioners of EOM methods in the chemistry community were $\mathrm{McKoy}^{8}$ and his group at Cal Tech. They imported many ideas and mathematical tools from the nuclear physics literature ${ }^{9}$, where EOM theories had been used to study excited states of nuclei, and they focused their efforts on electronic excitation energies $\Delta \mathrm{E}$, not IPs or EAs. In the early 1970 s, the author used the framework of EOM theory ${ }^{10}$ as expressed by the 
McKoy group to develop a systematic (i.e., order-by-order in the MøllerPlesset perturbation theory sense) approach for directly computing molecular EAs and IPs as eigenvalues of the EOM working equations. It is this development and its subsequent improvement and extensions ${ }^{11}$ by our group and others that we now describe.

\section{BASICS OF EOM THEORY}

\section{The EA Equations of Motion}

The fundamental working equations of any EOM theory can be derived by writing the Schrödinger equations for the neutral and anion (or neutral and cation or ground and excited) states of interest and subtracting the two equations as a first step toward obtaining a single equation that will yield the EA or IP or $\Delta \mathrm{E}$. That is, the EOM theory produces the intensive energy difference directly as an eigenvalue of the working equation. As above, we use $|0, N\rangle$ to denote the 0 -th electronic state of the $\mathrm{N}$-electron neutral and $|\mathrm{K}, \mathrm{N}+1\rangle$ to denote the $\mathrm{K}$-th state of the $\mathrm{N}+1$-electron anion and write the two Schrödinger equations as

$$
\begin{gathered}
H|0, N\rangle=E(0, N)|0, N\rangle \\
H|K, N+1\rangle=E(K, N+1)|K, N+1\rangle .
\end{gathered}
$$

Because $|0, N\rangle$ and $|K, N+1\rangle$ contain different numbers of electrons, it is convenient in developing EOM theories of EAs to express the electron Hamiltonian $\mathrm{H}$ in second-quantized form ${ }^{12}$ :

$$
H=\Sigma_{i, j} h(i, j) i+j+1 / 2 \Sigma_{i, j, k, l}\langle i, j \mid k, l\rangle i+j+1 k,
$$

where $h(i, j)$ represents a matrix element of the one-electron operators (i.e., kinetic energy, electron-nuclear Coulomb attraction, etc.) within the orthonormal molecular spin-orbital basis $\left\{\phi_{j}\right\}_{1}\langle i, j \mid k, I\rangle$ is a matrix element of the two-electron operators (i.e., electron-electron repulsion), and the set of Fermion creation operators $\left\{\mathcal{I}^{+}\right\}$create an electron in the $\left\{\phi_{i}\right\}$ spin-orbitals, whereas the \{\} \}operators destroy such an electron. Writing $\mathrm{H}$ in such a form 
allows us to use the same $H$ in Eqs (2a) and (2b) even those these two Schrödinger equations relate to $\mathrm{N}$ and $\mathrm{N}+1$ el ectrons, respectively.

The next step in developing an EOM equation is to assume that the anion state $|K, N+1\rangle$ can be related to the neutral state $|0, N\rangle$ through an operator $\mathrm{Q}^{+}(\mathrm{K})$ :

$$
|K, N+1\rangle=Q^{+}(K)|0, N\rangle
$$

that maps the neutral molecule wave function into the desired anion wave function. For the EA case at hand, the operator $\mathrm{Q}^{+}(\mathrm{K})$ is usually written in terms of scalar coefficients $t(K, I)$ multiplied by operators $T^{+}(I)$, also expressed in second-quantization language, each of which involves adding an electron

$$
Q^{+}(K)=\Sigma_{\mid} t(K, I) T^{+}(I)
$$

Manne showed ${ }^{13}$ that a complete set of such $T^{+}(I)$ operators consists of the union of sets of operators $\left\{p^{+}\right\}$that add an electron to a spin-orbital $\phi_{p}$, operators $\left\{p^{+} q^{+} a\right\}$ that add an electron to $\phi_{p}$ and excite another el ectron from $\phi_{a}$ to $\phi_{q}$, operators $\left\{p^{+} q^{+} r^{+} a b\right\}$ that add an electron to $\phi_{p}$ excite an electron from $\phi_{a}$ to $\phi_{\mathrm{r}}$ and excite another electron from $\phi_{\mathrm{b}}$ to $\phi_{\mathrm{q}}$ as well as higher-level electron addition and excitation operators up to the highest-level operators that add an electron and induce $\mathrm{N}$ excitations. In labeling these operators, the indices $a, b, c, d$, etc. are used to denote spin-orbitals occupied in a so-called reference Slater determinant within $|0, N\rangle$ and $p, q, r, s$, etc. are used to denote unoccupied (i.e., virtual) spin-orbitals. The reference determinant, which is what defines the concept of occupied and unoccupied spin-orbitals, is usually chosen to be the determinant $|0\rangle$ within the neutralmolecule wave function

$$
\left.|0, N\rangle=\Sigma_{J=0, M} C(0, J) \|\right\rangle
$$

with the largest amplitude $\mathrm{C}(0,0)$, but it has been shown ${ }^{13}$ that $|0\rangle$ can actually be taken to be any determinant within $|0, N\rangle$ that possesses non-zero amplitude. Later we will deal with how one determines the $\mathrm{C}(0, \mathrm{~J})$ amplitudes in the wave function $|0, N\rangle$; for now, suffice it to say these amplitudes 
can, for example, be taken from Møller-Plesset (MP) perturbation theory, from multiconfiguration self-consistent field (MC-SCF) theory, from configuration interaction $(\mathrm{Cl})$ theory or from coupled-cluster $(\mathrm{CC})$ theory.

Using Eq. (4) in Eqs (2) and subtracting Eq. (2a) from (2b) gives a single equation whose eigenvalue gives the desired EA:

$$
\left(H Q^{+}(K)-Q^{+}(K) H\right)|0, N\rangle=(E(K, N+1)-E(0, N)) Q^{+}(K)|0, N\rangle
$$

or, in terms of the commutator $\left[\mathrm{H}, \mathrm{Q}^{+}(\mathrm{K})\right]$

$$
\left[H, Q^{+}(K)\right]|0, N\rangle=E Q^{+}(K)|0, N\rangle
$$

where the eigenvalue $\mathrm{E}$ is the negative of the $\mathrm{EA}$. The key point is that one now has a single equation to be solved that produces the intensive EA as its eigenvalue. This equation appears to be of the conventional eigenvalueeigenfunction form, but it is somewhat different because the operator that acts on the eigenfunction $\mathrm{Q}^{+}(\mathrm{K})|0, \mathrm{~N}\rangle$ is not the Hamiltonian but a commutator involving the Hamiltonian. The fact that the commutator appears is what allows the eigenvalue to be an intensive energy difference.

To progress further toward practical implementation, specific choices must be made for how one is going to approximate the neutral-molecule wave function $|0, N\rangle$ and at what level one is going to truncate the expansion of the operator $\mathrm{Q}^{+}(\mathrm{K})$ given in Eq. (5). It is also conventional to reduce Eq. (7) to a matrix eigenvalue equation by projecting this equation onto an appropriately chosen space of $\mathrm{N}+1$-electron functions. Let us first deal with the latter issue.

Once the number of $\mathrm{T}^{+}(\mathrm{I})$ operators used to construct $\mathrm{Q}^{+}(\mathrm{K})$ has been chosen (we discuss this choice later), the total number Imax of $t(K, I)$ amplitudes has been determined. Multiplying Eq. (7) on the left by the adjoint $T(j)$ of any one of the $\mathrm{T}^{+}$operators, and then projecting the resultant equation against $\langle 0, N|$ gives one form of the working EOM EA equations:

$$
\Sigma_{\mid}\left\langle 0, N\left|T(j)\left[H, T^{+}(I)\right]\right| 0, N\right\rangle t(K, I)=E \Sigma_{l}\left\langle 0, N\left|T(j) T^{+}(I)\right| 0, N\right\rangle t(K, I) .
$$

To make use of this equation, the $\left\langle 0, N\left|T(j)\left[H, T^{+}(I)\right]\right| 0, N\right\rangle$ and $\left\langle 0, N\left|T(j) T^{+}(I)\right| 0, N\right\rangle$ matrices of dimension $I^{\max } \times I^{\text {max }}$ must first be evaluated in terms of one and 
two-electron integrals (appearing in $\mathrm{H}$ ) and one-, two-, and higher-body density matrices (depending upon the level at which the $\{T+(I)\}$ operator expansion is truncated). Subsequently, the EA values (i.e., EAs for the various anion states, $K$, relative to the $|0, N\rangle$ state of the neutral) are computed as minus the eigenvalues $\mathrm{E}$ of $\mathrm{Eq}$. (8).

The Analogous Equations of Motion for Ionization Potentials

It is useful to explore how this same framework has been used to compute molecular ionization potentials (IPS). It is fairly straightforward to show that an equation analogous to Eq. (7) but reading

$$
\langle 0, N|\left(H Q^{+}(K)-Q^{+}(K) H\right)=(E(0, N)-E(K, N-1))\langle 0, N| Q^{+}(K)
$$

is valid if the operators $\left\{\mathrm{Q}^{+}(\mathrm{K})\right\}$ are as given in Eq. (5) but with the $\left\{T^{+}(\mathrm{I})\right\}$ defined to include operators of the form $\left\{a^{+}, a^{+} b^{+} p, a^{+} b^{+} c^{+} q r\right.$, etc. $\}$. Of course, in Eq. (9), the operators within $\mathrm{Q}^{+}(\mathrm{K})$ act to the left on $\langle 0, N|$ to generate cationic states. As a result, neutral-cation energy differences appear in Eq. (9) and thus this offers a route to computing IPs. Multiplying this equation on the right by any one of the $T(j)$ operators and then projecting against $|0, N\rangle$ gives

$$
\Sigma_{\mid}\left\langle 0, N\left|\left[H, T^{+}(I)\right] T(j)\right| 0, N\right\rangle t(K, I)=E \Sigma_{1}\left\langle 0, N\left|T^{+}(I) T(j)\right| 0, N\right\rangle t(K, I)
$$

but now the eigenvalues $E$ denote values of $(E(0, N)-E(K, N-1))$, which are the negatives of the IPS.

Thus far, we see that EOMs can be written that allow EAs or IPs to be computed. The fundamental constructs within these equations are as follows:

1. for the EA case, matrix elements $\left\langle 0, N\left|T(j)\left[H, T^{+}(I)\right]\right| 0, N\right\rangle$ involving the commutator of $\mathrm{H}$ with the $\mathrm{T}^{+}(\mathrm{I})$ operators then multiplied on the left by a $\mathrm{T}(\mathrm{j})$ operator, as well as an analogous overlap matrix element $\left\langle 0, N\left|T(j) T^{+}(I)\right| 0, N\right\rangle$;

2. for the IP case, matrix elements $\left\langle 0, N\left|\left[H, T^{+}(I)\right] T(j)\right| 0, N\right\rangle$ of the same commutator but with the $\mathrm{T}(\mathrm{j})$ operator on the right, as well as the corre sponding overlap matrix element $\left\langle 0, N\left|T^{+}(I) T(j)\right| 0, N\right\rangle$;

3. the neutral-molecule wave function $|0, N\rangle$ with respect to which the EA or IP is to be evaluated. 
The Rank of the Operators

It is now useful to analyze the density matrix elements ${ }^{14}$ that enter into these equations. Each of the $T^{+}(\mathrm{j})$ operators contains an odd number of creation or annihilation operators, and the Hamiltonian $\mathrm{H}$ contains two (i.e., $i+j$ ) or four (i.e., $i^{+} j+k$ ) such operators. It can be seen that the commutator $\left[H, T^{+}(I)\right]$ does not contain four plus the number of creation or annihilation operators in $T^{+}(I)$, but two fewer operators. For example, the commutator $\left[i+j+1 k, p^{+} q^{+} a\right]$ does not yield any terms with four creation and three annihilation operators but only terms with three creation and two annihilation operators. We say that the act of forming the commutator (which is what causes the higher-order operators to cancel) gives rise to a reduction in the rank of the operators. As a result, both the operator products $T(j)\left[H, T^{+}(I)\right]$ and $\left[H, T^{+}(I)\right] T(j)$, which appear in the EA and IP equations of motion, respectively, contain terms only involving both creation and annihilation operators equal to the number of creation operators in $T^{+}(I)$ plus one plus the number of creation operators in $T(j)$. For example, if $T^{+}(I)=p^{+} q^{+} a$ and $\mathrm{T}(\mathrm{j})=\mathrm{b}^{+} \mathrm{rs}$, then $\mathrm{T}(\mathrm{j})\left[\mathrm{H}, \mathrm{T}^{+}(\mathrm{I})\right]$ and $\left[\mathrm{H}_{,} \mathrm{T}^{+}(\mathrm{I})\right] \mathrm{T}(\mathrm{j})$ will contain terms with no more than four creation and four annihilation operators. This means that the density matrices needed to from $\left\langle 0, N\left|T(j)\left[H, T^{+}(I)\right]\right| 0, N\right\rangle$ and $\left\langle 0, N\left|\left[H, T^{+}(I)\right] T(j)\right| 0, N\right\rangle$ will be, at most, fourth-order density matrices of the $\langle 0, N|\ldots| 0, N\rangle$ density.

Equations of Lower Rank for Both EAs and IPS

Indeed, in the early years of using EOM methods ${ }^{15}$ to compute EAs and IPS, operator manifolds of the form $\left\{T^{+}(I)\right\}=\left\{p^{+} ; p^{+} q^{+} a, p^{+} q^{+} r^{+} b\right.$ a, etc. $\}$ or $\left\{T^{+}(I)\right\}=$ $\left\{a^{+}, a^{+} b^{+} p, a^{+} b^{+} c^{+} q r\right.$, etc. $\}$ were employed with MP approximations to $|0, N\rangle$ (usually taken through first order) to form the kind of matrix el ements appearing in Eqs (8) and (10) and to then evaluate EAs and IPs from their eigenvalues $\mathrm{E}$. However, it became more common to use a combination of the EA and IP EOMs formed by adding Eqs (8) and (10), while expanding the $\left\{T^{+}(I)\right\}$ operator manifold to include both those needed to evaluate EAs $\left\{p^{+} ; p^{+} q^{+} a, p^{+} q^{+} r^{+} b a\right.$, etc. $\}$ and those needed for the IPs $\left\{a^{+}, a^{+} b^{+} p, a^{+} b^{+} c^{+} q r\right.$, etc. \}, to simultaneously compute both such energy differences.

To understand why such a combination has proven beneficial, it suffices to examine the form and rank of the operators whose $\langle 0, N|\ldots| 0, N\rangle$ matrix elements must be evaluated 


$$
\begin{aligned}
& \Sigma_{\mid}\left\langle 0, N\left|\left[H, T^{+}(I)\right] T(j)+T(j)\left[H, T^{+}(I)\right]\right| 0, N\right\rangle t(K, I)= \\
& =E \Sigma_{\mid}\left\langle 0, N\left|T^{+}(I) T(j)+T(j) T^{+}(I)\right| 0, N\right\rangle t(K, I) .
\end{aligned}
$$

Recall that the $\mathrm{T}^{+}(\mathrm{j})$ operators contain an odd number of creation or annihiIation operators. Each of the products $\left[H, T^{+}(I)\right] T(j), T(j)\left[H, T^{+}(I)\right], T^{+}(I) T(j)$, and $T(j) T^{+}(I)$ thus contain an even number of such operators. However, because of the fundamental anti-commutation properties of these operators

$$
\begin{gathered}
i^{+} j+j i^{+}=\delta_{i, j} \\
i j+j i=0 \\
i^{+} j^{+}+j^{+} i^{+}=0
\end{gathered}
$$

it can easily be shown that the operator combinations $T^{+}(\mathrm{I}) \mathrm{T}(\mathrm{j})+\mathrm{T}(\mathrm{j}) \mathrm{T}^{+}(\mathrm{I})$ and $\left[H, T^{+}(I)\right] T(j)+T(j)\left[H, T^{+}(I)\right]$ contain one fewer creation and one fewer annihilation operator than does either of the two terms in the sums. So, by combining the EA and IP EOMs, one effects an additional rank reduction in the operators appearing in the equations although the dimensions of the matrices one needs to construct are doubled (because the $\left\{T^{+}(I)\right\}$ operator manifold is doubled) when both EA and IP operators were included. The rank reduction is important because it means that the density matrices that need to be evaluated to compute the $\langle 0, N|\ldots| 0, N\rangle$ matrix elements are of lower rank in Eq. (11) than in either Eq. (8) or (10). As we said, it has become more common to use the combined EA and IP Eq. (11) because lower-order density matrices are required.

\section{Summary}

Thus far, we have shown how one can obtain eigenvalue equations, in which the energy eigenvalues correspond to the intensive EAs (or IPs), by postulating that the anion (or cation) wave function can be related to the neutral-molecule wave function through an operator. We have also shown how the EA and IP equations of motion can be combined to generate a combined EOM from which both EAs and IPs can be obtained. The advan- 
tage to the latter approach is that the operators appearing in the resultant equations are of lower rank and thus lower-order density matrices must be evaluated to carry out the calculations. Let us now move on to address more specific embodiments of such EOM theories that result from different choices of the neutral-molecule wave function and of the operator connecting the neutral and anion wave functions.

\section{PRACTICAL IMPLEMENTATIONS OF EOM THEORIES FOR EAS AND IPS}

The basic ideas underlying any EOM method for computing EAs or IPS appear above. However, as discussed earlier, in any specific embodiment of such a method, one must commit to (i) a specific approximation to the neutral-molecule wave function $|0, N\rangle$, (ii) a specific choice of how large an operator manifold $\{T+(I)\}$ to employ, and (iii) how to solve the resultant EOM equations for the eigenvalues $E$ that then produce the EAs or IPs. In the following subsections, we describe the most commonly used choices for these three issues.

The Møller-Plesset-Based Approximations

In the earliest implementation of EOM approaches to EAs, the author's group $^{10,15}$ chose to represent the $|0, N\rangle$ wave function in MP expansion

$$
|0, N\rangle=\psi^{0}+\psi^{1}+\psi^{2}+\ldots
$$

with the single-determinant unrestricted Hartree-Fock (HF) function being $\psi^{0}$ and the corresponding neutral-molecule $\mathrm{HF}$ Hamiltonian being $\mathrm{H}^{0}$. This choice was made because there existed substantial evidence that EAs and IPs computed at the Koopmans' theorem level would not meet the desired $0.1 \mathrm{eV}$ accuracy. The evidence on atoms and small molecules also showed that EAs and IPs computed using standard second-order MP theory were much more accurate but not sufficient to approach the $0.1 \mathrm{eV}$ standard. For this reason, the author's group set their sites on the next reasonable level, that of third-order MP theory and by expanding $|0, N\rangle$ in the MPn series, we were able to properly keep track of the orders of various contributions to the working equations.

The operator manifold $\left\{T^{+}(I)\right\}$ was taken to consist of $\left\{p^{+} ; p^{+} q^{+} a\right\}$ and $\left\{a^{+}, a^{+} b^{+} p\right\}$ because this choice of operator manifold was shown to be capable of producing 
EAs and IPs that were precise through third order6o in the MP perturbation, which is why this choice was made.

The resultant variant of Eq. (11) was not solved by finding the eigenvalues of this matrix eigenvalue equation whose dimension is the sum of the dimensions of the $\left\{p^{+} ; p^{+} q^{+} a\right\}$ and $\left\{a^{+}, a^{+} b^{+} p\right\}$ operator manifolds. Rather, that large matrix eigenvalue problem was partitioned ${ }^{10}$ using a primary subspace defined by the $\left\{p^{+}, a^{+}\right\}$operators and a secondary subspace defined by the $\left\{p^{+} q^{+} a, a^{+} b^{+} p\right\}$ operators. This choice was made because the class of anion wave functions we wanted to study were those described, at the lowest level, by adding an electron to a virtual orbital of the neutral. The partitioned eigenvalue problem

$$
\Sigma_{j=a, p} H_{i, j}(E) X_{j}=E X_{i},
$$

whose dimension is that of the $\left\{p^{+}, a^{+}\right\}$operator space was used to find the eigenvalues $\mathrm{E}$. Of course, the act of partitioning the higher-dimension matrix eigenvalue problem does not change the values of $E$ that represent solutions to the equations. That is, the same $E$ values that fulfil the original equations are also solutions to the partitioned equations. However, once one introduces approximations designed to evaluate elements of the partitioned $\mathrm{H}_{\mathrm{i}, \mathrm{j}}(\mathrm{E})$ matrix to a chosen order in perturbation theory, this equivalence is lost. It is precisely by making such an order analysis (e.g., computing $\mathrm{H}_{\mathrm{i}, \mathrm{j}}(\mathrm{E})$ through second or third order) that EOM theories capable of evaluating EAs or IPs to a given order were obtained.

When the elements of the partitioned matrices were evaluated through second order in the MP series, the following expression was obtained for the matrix elements $H_{i, j}$ :

$$
\begin{gathered}
\mathrm{H}_{\mathrm{i}, \mathrm{j}}(\mathrm{E})=\varepsilon_{\mathrm{i}} \delta_{\mathrm{i}, \mathrm{j}}-\Sigma_{\mathrm{p}, \mathrm{q}, \mathrm{a}}\langle\mathrm{i}, \mathrm{a}|| \mathrm{p}, \mathrm{q}\rangle\langle\mathrm{p}, \mathrm{q} \mid \mathrm{lj}, \mathrm{a}\rangle /\left(\varepsilon_{\mathrm{p}}+\varepsilon_{\mathrm{q}}-\varepsilon_{\mathrm{a}}-\mathrm{E}\right)+ \\
+\Sigma_{\mathrm{a}, \mathrm{b}, \mathrm{p}}\langle\mathrm{i}, \mathrm{p} \| \mathrm{a}, \mathrm{b}\rangle\langle\mathrm{a}, \mathrm{b} \mid \mathrm{j}, \mathrm{p}\rangle /\left(\varepsilon_{\mathrm{a}}+\varepsilon_{\mathrm{b}}-\varepsilon_{\mathrm{p}}-\mathrm{E}\right) .
\end{gathered}
$$

Here, the $\varepsilon_{\mathrm{i}}$ denote the UHF spin-orbital energies of the neutral molecule and the $\langle i, j|| k, I\rangle$ denote differences in two-electron integrals $(\langle i, j|| k, I\rangle=$ $\langle i, j \mid k, I\rangle-\langle i, j \mid l, k\rangle)$. Such expressions were obtained earlier by Reinhardt and Doll ${ }^{6 n}$ within the Greens function framework, but they had not extended their efforts to third or higher orders as the author's group was aiming for. 
The expression for $\mathrm{H}_{\mathrm{i}, \mathrm{j}}(\mathrm{E})$ valid through third order in the MP series is more complicated and is derived in lit. ${ }^{60,10}$ where the partitioned matrix eigenvalue equation was written as follows:

\section{$H(E) X=E X$,}

where the elements of the $\mathbf{H}$ matrix are defined as follows:

$$
\begin{gathered}
H_{i, j}(E)=A_{i, j}+\sum_{\alpha_{<} \beta, m}\left[B_{i, \alpha_{m} \beta} B *_{j, \alpha_{m} \beta}\right] /\left[E_{\alpha \beta}^{m}+E\right]- \\
-\sum_{N+1<n, \alpha}\left[B_{i, n} \alpha_{N+1} B *_{j, n} \alpha_{N+1}\right] /\left[E_{\alpha}^{N+1 n}-E\right]-\Sigma_{N+1<m<n, \alpha}\left[B_{i, n \alpha_{m}} B *_{j, n} \alpha_{m}\right] /\left[E_{\alpha}^{m n}-E\right] .
\end{gathered}
$$

In turn, the elements of the $\mathbf{A}$ and $\mathbf{B}$ matrices are shown below:

$$
\begin{aligned}
& B_{i, \alpha_{m} \beta}=-\langle i m \mid \alpha \beta\rangle-1 / 2 \Sigma_{p, q}\langle i m \mid p q\rangle K_{(\alpha \beta)}{ }^{(p q)}+ \\
& +\Sigma_{\gamma, p}\left[\langle i \gamma \mid p \alpha\rangle K_{(\beta \gamma)}{ }^{(m p)}-\langle i \gamma \mid p \beta\rangle K_{(\alpha \gamma)}{ }^{(m p)}\right] \\
& B_{i, n \alpha_{m}}=-\langle i \alpha \mid m n\rangle-1 / 2 \Sigma_{\gamma, \delta}\langle i \alpha \mid \gamma \delta\rangle K_{(\delta \gamma)}{ }^{(m n)}+ \\
& +\Sigma_{\gamma, p}\left[\langle i p \mid \gamma n\rangle K_{(\alpha \gamma)}{ }^{(m p)}-\langle i p \mid \gamma m\rangle K_{(\alpha \gamma)}{ }^{(n p)}\right] \\
& A_{i, j}=\delta_{i, j} \varepsilon_{i}+\Sigma_{k, l}\langle i k \mid j l\rangle F_{k l}
\end{aligned}
$$

to which one adds the following E-independent terms

$$
\begin{aligned}
\delta A_{i, j}= & \Sigma_{\delta, \beta, p, m, n}\langle j p \| i \delta\rangle\langle\delta \beta \| m n\rangle\langle m n \| p \beta\rangle /\left[\left(\varepsilon_{\delta}-\varepsilon_{p}\right)\left(\varepsilon_{\delta}+\varepsilon_{\beta}-\varepsilon_{m}-\varepsilon_{n}\right)\right]+ \\
& +\Sigma_{\delta, \beta, p, m, n}\langle j \delta \| i p\rangle\langle p \beta \| m n\rangle\langle m n \| \delta \beta\rangle /\left[\left(\varepsilon_{\delta}-\varepsilon_{p}\right)\left(\varepsilon_{\delta}+\varepsilon_{\beta}-\varepsilon_{m}-\varepsilon_{n}\right)\right]+ \\
& +\Sigma_{\delta, \alpha, \beta, p, n}\langle j p \| i \delta\rangle\langle\delta n \| \beta \alpha\rangle\langle\alpha \beta \| p n\rangle /\left[\left(\varepsilon_{\delta}-\varepsilon_{p}\right)\left(\varepsilon_{\alpha}+\varepsilon_{\beta}-\varepsilon_{p}-\varepsilon_{n}\right)\right]+ \\
& +\Sigma_{\delta, \alpha, \beta, p, n}\langle j \delta \| i p\rangle\langle\delta n \| \beta \alpha\rangle\langle\alpha \beta \| p n\rangle /\left[\left(\varepsilon_{\delta}-\varepsilon_{p}\right)\left(\varepsilon_{\alpha}+\varepsilon_{\beta}-\varepsilon_{p}-\varepsilon_{n}\right)\right] .
\end{aligned}
$$

The energy denominators appearing in the $\mathrm{H}_{\mathrm{ij}}$ matrix elements are 


$$
\begin{aligned}
& E_{\alpha}^{m n}=\varepsilon_{m}+\varepsilon_{n}-\varepsilon_{\alpha}-\langle\alpha m \mid \alpha m\rangle-\langle\alpha n \mid \alpha n\rangle+\langle m n \mid m n\rangle \\
& E_{\delta \gamma}^{p}=\varepsilon_{p}+\varepsilon_{\delta}-\varepsilon_{\gamma}-\langle\delta p \mid \delta p\rangle-\langle\gamma p \mid \gamma p\rangle+\langle\delta \gamma \mid \delta \gamma\rangle .
\end{aligned}
$$

Finally, the $\mathrm{F}$ quantities appearing above are given as

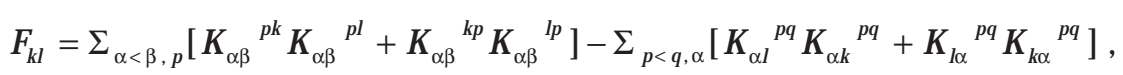

where

$$
\begin{gathered}
K_{\alpha \beta}{ }^{(p q)}=K_{\alpha \beta}^{p q}-K_{\alpha \beta}{ }^{q p} \\
K_{(\alpha \beta)}^{p q}=K_{\alpha \beta}^{p q}-K_{\beta \alpha}^{p q} \\
K_{(\alpha \beta)}{ }^{p q q)}=K_{\alpha \beta}^{p q}-K_{\alpha \beta}{ }^{q p}-K_{\beta \alpha}^{p q}+K_{\beta \alpha}{ }^{q p},
\end{gathered}
$$

and the latter quantities are the MP expansion coefficients of the first-order wave function:

$$
\mathrm{K}_{\alpha \beta}{ }^{\mathrm{mn}}=\langle\mathrm{mn} \mid \alpha \beta\rangle /\left(\varepsilon_{\alpha}+\varepsilon_{\beta}-\varepsilon_{\mathrm{m}}-\varepsilon_{\mathrm{n}}\right) .
$$

Although more complicated than their second-order counterparts, the basic structure of the above expressions for $\mathrm{H}_{i, j}(E)$ are the same as those in second order.

These third-order equations have been used in many applications in which molecular EAs have been computed for a wide variety of species as illustrated in lit. ${ }^{15}$. Clearly, all of the quantities needed to form the second- or third-order EOM matrix elements $\mathrm{H}_{\mathrm{j}, \mathrm{k}}$ are ultimately expressed in terms of the orbital energies $\left\{\varepsilon_{\mathrm{k}}\right\}$ and two-electron integrals $\langle\mathrm{j}, \mathrm{k} \mid \mathrm{I}, \mathrm{h}\rangle$ evaluated in the basis of the neutral molecule's HF orbitals that form the starting point of the MP theory. However, as with most electronic structure theories, much effort was subsequently devoted to recasting the working EOM equations in a manner that involves the atomic-orbital (AO) two-electron integrals rather than the molecular-orbital based integrals. Because such technical matters of direct AO-driven calculations are outside the scope of this 
work, we will not delve into them further here although we note that Oddershede ${ }^{16}$ in collaboration with our group looked into how to express EOM-type calculations in the AO basis.

\section{Relationship to Greens Functions/Propagators}

It turns out that in the early 1970s when we were developing and imple menting the EOM method for treating EAs and IPS, several groups had taken a different approach to the evaluation of atomic and molecular electronic energy differences using what were called Greens functions (GFs) or propagators. Linderberg and Öhrn pioneered ${ }^{6 p}, 6 y$ the use of such methods in quantum chemistry, while Cederbaum and co-workers ${ }^{6 q}, 6 r, 6 s$, Reinhardt and Doll ${ }^{6 n}$, Taylor, Yaris, and co-workers ${ }^{6 \mathrm{t}}$ and Pickup and Goscinski6u were among the first to apply the methods to EAs and IPs using an ab initio approach. Purvis and Öhrn ${ }^{6 v, 6 w}$ soon thereafter expanded the range of the the ory to include open-shell systems. These workers as well as Jørgensen and Oddersh ede $\mathrm{e}^{6 \mathrm{~b}}$ and others ${ }^{4 \mathrm{~h}, 6 \mathrm{c}, 6 \mathrm{f}, 6 \mathrm{~g}, 6 \mathrm{i}, 6 \mathrm{j}, 6 \mathrm{k}}$ developed MP-based GFs for evaluating electronic excitation energies but we will not discuss these developments further here because our emphasis is on IPS and EAs.

The GF EA and IP theories were derived from consideration of the following time-dependent matrix elements:

$$
\begin{aligned}
\mathrm{G}_{\mathrm{j}, \mathrm{k}}(\mathrm{t}) & =(\mathrm{l} / \mathrm{i} \hbar) \Theta(\mathrm{t})\left\langle 0, \mathrm{~N}\left|\exp (\mathrm{iH} \mathrm{t} / \hbar) \mathrm{j}^{+} \exp (-\mathrm{iH} \mathrm{t} / \hbar) \mathrm{k}\right| \mathrm{O}, \mathrm{N}\right\rangle+ \\
& +(\mathrm{l} / \mathrm{i} \hbar) \Theta(-\mathrm{t})\left\langle 0, \mathrm{~N}\left|\mathrm{k} \exp (\mathrm{iH} \mathrm{t} / \hbar) \mathrm{j}^{+} \exp (-\mathrm{iH} \mathrm{t} / \hbar)\right| 0, \mathrm{~N}\right\rangle .
\end{aligned}
$$

Here, $\Theta(t)$ is the Heaviside step function, which equals unity when t is positive and zero when $t$ is negative, $\mathrm{j}^{+}$and $\mathrm{k}$ are the same creation and annihilation operators discussed earlier, and $|0, N\rangle$ is the neutral-molecule reference wave function. Introducing complete sets of $\mathrm{N}-1$ and $\mathrm{N}+1$ electron Hamiltonian eigenfunctions into the first and second terms in Eq. (16), it is straightforward to see that one observes time dependences varying as $\exp (i[E(0, N)-E(K, N-1)] t / \hbar$ and $\exp (i[E(K, N+1)-E(0, N)] t / \hbar$, respectively.

Taking the time derivative of Eq. (16), one obtains expressions involving

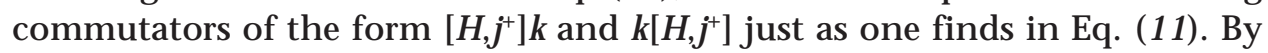
analyzing the resulting time-derivative equations, workers in this field were able to obtain equations that such $G_{j, k}(t)$ matrix elements obey (n.b., these were called the equations of motion for these matrix elements). The workers named above were able to express the resulting equations in terms of 
one and two-electron integrals and corresponding density matrices much as the author had done within the EOM framework. In fact, it turned out that the final working equations of the so-called one-electron GF or electron propagator defined in Eq. (16), when Fourier transformed from the time to the energy domain, were exactly the same as the EOM equations given above (i.e., Eq. (15) and those reproduced from lit. ${ }^{60,10}$ ). However, only the Cederbaum group achieved the full third-order expressions within the GF framework analogous to what we achieved from the EOM point of view as reviewed above.

Especially in recent years, much of the work aimed at calculating EAs and IPs using these direct-calculation EOM and GF methods has been performed within the notation of GF and has been carried out by Ortiz group ${ }^{17}$ as well as by the Cederbaum group. To further illustrate the impact that such advances have had within the quantum chemistry community, we note that the Ortiz group has implemented various (i.e., Møller-Plesset up to third-order and other) variants of these theories within the highly successful Gaussian ${ }^{18 a}$ suite of computer codes as a result of which many workers worldwide now employ EOM of GF-type methods to evaluate EAs and IPs. However, the extension of the working EOMs to higher than third order in the MPn series has not yet been made, at least not in a widely used framework. Instead, as we discuss later, alternative (i.e., nonperturbative) approximations to the function $|0, N\rangle$ have been used to extend the accuracy and range of applicability of EOM and GF methods.

\section{The Natural Orbital or Extended Koopmans' Theorem Approach}

In the mid 1970s, Parr and co-workers ${ }^{19}$ and, independently, Smith and co-workers ${ }^{20}$ proposed to use an equation such as Eq. (10) for computing IPs and they referred to these methods as natural-orbital or extended Koopmans' theorem theories. Subsequently, Andersen and the author ${ }^{21}$ analyzed the working equations of this approach through second and third order in the MP series and noted differences between them and the GF and equivalent EOM theories computed through these same orders. Of course, based on the discussion of the next Section, these differences relate to the ranks of the operators appearing in the working equations and are not surprising. More recently, Cioslowski and co-workers ${ }^{22}$ have shown that these extended Koopmans' theorem approaches indeed offer a very efficient and reasonably accurate route to computing IPS or EAs, so it is likely that these methods will continue to develop. One of the more attractive aspects of the extended Koopmans' approaches is that they have been shown ${ }^{23}$ to be 
capable, at least in principle, of giving the correct lowest ionization potential of a neutral molecule because they are capable of generating the proper asymptotic form for the electron density, which is known to vary as $\exp \left(-2(2 \mid \mathrm{IP})^{1 / 2} r\right)$.

\section{Multiconfiguration-Based Approximations}

Following on the proof by Manne $e^{13}$ that the operator spaces $\left\{T^{+}(I)\right\}=\left\{p^{+}\right.$; $p^{+} q^{+} a, p^{+} q^{+} r^{+} b a$, etc. $\}$ and $\left\{T^{+}(I)\right\}=\left\{a^{+}, a^{+} b^{+} p, a^{+} b^{+} c^{+} q r\right.$, etc. $\}$ can be used (i.e., are capable of forming complete sets of ion states) even when no single determinant forms a dominant component of the neutral-molecule wave function $|0, N\rangle$, the author's group extended the combined EA and IP EOM theory to the case in which $|0, N\rangle$ is of an arbitrary multiconfiguration self-consistent field (MC-SCF) form ${ }^{11 g}$ and the ionization operator manifold $\left\{T^{+}(I)\right\}$ included operators of the form $\left\{p^{+} ; p^{+} q^{+} a\right\}$ and $\left\{a^{+}, a^{+} b^{+} p\right\}$. The resultant working equations were written as in Eq. (14), with the $\mathrm{H}_{\mathrm{j}, \mathrm{k}}$ matrix elements given in Eqs (18) of lit. ${ }^{11 g}$, which we do not reproduce here because of their complexity. The primary additional difficulty involved in implementing these multiconfiguration-based equations is the fact that three-electron density matrices $\left\langle 0, N\left|i^{+} j^{+} k^{+}\right| \mathrm{h} n \mid 0, N\right\rangle$ taken with respect to the MC-SCF wave function $|0, N\rangle$ are involved. These density matrices arise when the commutators $\left[\mathrm{H}, \mathrm{p}^{+} \mathrm{q}^{+} \mathrm{a}\right]$ and $\left[\mathrm{H}, \mathrm{a}^{+} \mathrm{b}^{+} \mathrm{p}\right]$ are evaluated.

To date, not much use has been made of the MC-SCF-based EOM theories as developed in the author's group. Instead, the framework of timedependent response theory, which can treat essentially any kind of reference wave function $|0, N\rangle$ including the MC-SCF variety, has superseded the EOM-based developments for such cases. It is important to keep in mind, however, that both the EOM and response function theories involve formulating and solving sets of equations whose solution (i.e., the unknown energy) is an intensive energy and, as such, they are all members of the same family of methods.

\section{Coupled-Cluster-Based EOM}

Of course, the coupled-cluster framework that Prof. Paldus was a pioneer in developing ${ }^{24}$, has also been used as a basis for deriving equations of motion. The use of coupled-cluster (CC) wave functions within EOM theory for excitation energies, IPs, and EAs has been developed ${ }^{25,26}$ upon slightly different lines than outlined in previous Section. The CC wave function 
ansatz for $|0, N\rangle$ is written as usual in terms of an exponential operator acting on a single-determinant (e.g., unrestricted HF) "reference function" $|0\rangle$

$$
|0, N\rangle=\exp (T)|0\rangle
$$

The so-called cluster operator $T$ is expressed in terms of spin-orbital excitation operators of the form $\left\{T_{1}\right\}=\left\{p^{+} a\right\},\left\{T_{2}\right\}=\left\{p^{+} q^{+} b a\right\},\left\{T_{3}\right\}=\left\{p^{+} q^{+} r^{+} c b a\right\}$, etc. with $T_{k}$ relating to the excitation of $k$ electrons from occupied spin-orbitals $(a, b, c$, etc.) to virtual spin-orbitals ( $p, q, r$, etc.). Prior to forming any EA EOM, the neutral-molecule CC equations need to be solved for the amplitudes $\left\{t_{n}\right\}$ that multiply the $\left\{T_{n}\right\}$ operators to form the CC T operator. For completeness, let us briefly review how the conventional CC wave function evaluation is carried out.

We recall the CC equations are formed by manipulating the Schrödinger equation

$$
H \exp (T)|0\rangle=E \exp (T)|0\rangle
$$

to read

$$
\exp (-T) H \exp (T)|0\rangle=E|0\rangle
$$

and subsequently projecting this equation against the set of functions $\left\{\langle 0| T_{n}{ }^{+}\right\}$. Because the $T$ operator contains only creation operators for unoccupied spin-orbitals and annihilation operators for occupied spin-orbitals, it turns out that the commutator expansion

$$
\begin{aligned}
\exp (-T) H \exp (T)= & H-[T, H]+1 / 2[T,[T, H]]-1 / 3 ![T,[T,[T, H]]]+ \\
& +1 / 4 ![T,[T,[T,[T, H]]]]+\ldots
\end{aligned}
$$

exactly truncates at the fourth-order term. So, the final working equations of CC theory can be written as 


$$
\begin{gathered}
\langle 0| T_{n}^{+}\{H-[T, H]+1 / 2[T,[T, H]]-1 / 3 ![T,[T,[T, H]]]+ \\
+1 / 4 ![T,[T,[T,[T, H]]]]\}|0\rangle=0
\end{gathered}
$$

Once the CC amplitudes $\left\{t_{n}\right\}$ are determined by solving these quartic equations, the CC energy is computed as

$$
\begin{gathered}
\langle 0| H-[T, H]+1 / 2[T,[T, H]]-1 / 3 ![T,[T,[T, H]]]+ \\
+1 / 4 ![T,[T,[T,[T, H]]]]|0\rangle=E
\end{gathered}
$$

The operator $\mathrm{Q}^{+}(\mathrm{K})$ that maps the $\mathrm{CC}$ wave function $|0, \mathrm{~N}\rangle$ into an anion or cation state is expressed as in Eq. (5) with the $\left\{T^{+}(I)\right\}$ operators including, for example, $\left\{T^{+}(I)\right\}=\left\{p^{+} ; p^{+} q^{+} a, p^{+} q^{+} r^{+} b\right.$ a, etc. $\}$ when EAs are to be computed and the adjoints of $\left\{a^{+}, a^{+} b^{+} p, a^{+} b^{+} c^{+} q r\right.$, etc. $\}$ when IPs are computed. The basic EOM analogous to Eq. (7b) is then written as

$$
\left[H, Q^{+}(K)\right] \exp (T)|0\rangle=E Q^{+}(K) \exp (T)|0\rangle
$$

Multiplying on the left by $\exp (-\mathrm{T})$ and realizing that $\mathrm{T}$ and $\mathrm{Q}^{+}(\mathrm{K})$ commute reduces this equation to

$$
\left[H^{\prime}, Q^{+}(K)\right]|0\rangle=E Q^{+}(K)|0\rangle
$$

where

$$
H^{\prime}=\exp (-T) H \exp (T) \text {, }
$$

which can be expanded as in Eq. (20) to involve at most quartic terms in the $\left\{_{n}\right\}$ amplitudes. Then, multiplying on the left by $\langle 0| T(j)$ reduces the EOM equations to their final working form

$$
\Sigma_{\mid}\langle 0| T(j)\{H-[T, H]+1 / 2[T,[T, H]]-1 / 3 ![T,[T,[T, H]]]+
$$$$
+1 / 4 ![T,[T,[T,[T, H]]]]\}, T^{+}(I)|0\rangle t(K, I)=E \Sigma_{1}\left\langle 0\left|T(j) T^{+}(I)\right| 0\right\rangle t(K, I) \text {. }
$$ 
This set of matrix eigenvalue equations is then solved to obtain $E$ which gives the EA or the IP (depending on what operator set was used). Such so-called electron-attached and electron-removed equations of motion (EA-EOM and IP-EOM) approaches have proven highly successful 25,26 in computing EAs and IPs of a wide range of atoms and molecules primarily because the coupled-cluster treatment of electron correlation provides such a highly accurate treatment of the dynamical electron correlation. At present there is a great deal of activity within this framework of utilizing EOM theories for computing EAs, IPS, and $\Delta \mathrm{Es}$.

It should be noted that Eq. (26) does not contain an E-dependent matrix element on its left-hand side as do the MPn EOM equations (Eq. (14)) or the equivalent GF equations. This is because the EOM-CC equations are usually not subjected to the operator-space partitioning that the GF and MPn-based EOM theories commonly employ. It should also be noted that the operators appearing on the left-hand side of Eq. (26) produce nonHermitian matrices. As a result, there arise non-orthogonal left- and righteigenvectors in solving Eq. (26). As is discussed 25,26 , it is important to compute both sets of eigenvectors if one wishes to compute, for example, photoelectron intensities.

\section{METASTABLE ANION STATES}

A special kind of problem arises when one attempts to compute the EA of a molecule whose anion is not electronically bound relative to the corresponding neutral. For example, the $X^{1} \Sigma_{g}{ }^{+}$state of the $\mathrm{N}_{2}$ molecule does not bind an electron to form an electronically stable anion. Instead the $X^{2} \Pi_{g}$ state of $\mathrm{N}_{2}{ }^{-}$, formed by adding an electron to the $\pi_{\mathrm{g}}$ anti-bonding orbital of $\mathrm{N}_{2}$ is a so-called resonance state that lies higher in energy than $\mathrm{N}_{2}$ and can spontaneously eject its excess electron. One cannot simply employ conventional basis sets and $a b$ initio electronic structure methods (including EOM or GF or response-function methods) to correctly determine the energies of such states.

The most common and powerful tool for studying such metastable states theoretically is the so-called stabilization method (SM). This method, pioneered by Taylor's group ${ }^{27}$, involves embedding the system of interest (e.g., the $\mathrm{N}_{2}^{-1}$ anion) within a finite "box" in order to convert the continuum of states corresponding, for example, to $\mathrm{N}_{2}+\mathrm{e}$, into discrete states that can be handled by conventional square-integrable basis functions using, for example, the EOM method. By varying the size of the box, one can vary the energies of the discrete states that correspond to $\mathrm{N}_{2}+$ e (i.e., one varies the 
box size to vary the kinetic energy KE of the orbitals containing the excess electron). As the box size is varied, one eventually notices (e.g., by plotting the orbitals) that one of the $\mathrm{N}_{2}+$ e states obtained in the EOM process possesses a significant amount of valence character. That is, one such state has significant amplitude not only at large $r$ but also in the region of the two nitrogen centers. It is this state that corresponds to the metastable resonance state, and it is the EOM eigenvalue E of this state that provides the stabilization estimate of the resonance state energy relative to that of the neutral $\mathrm{N}_{2}$.

Let us continue using $\mathrm{N}_{2}^{-1}$ as an example for how one usually varies the box within which the anion is constrained. One uses a conventional atomic orbital basis set that likely includes $\mathrm{s}$ and $\mathrm{p}$ functions on each $\mathrm{N}$ atom, perhaps some polarization $d$ functions and some conventional diffuse $s$ and $p$ orbitals on each $\mathrm{N}$ atom. These basis orbitals serve primarily to describe the motions of the electrons within the usual valence regions of space. To this basis, one appends an extra set of diffuse $\pi$-symmetry orbitals. These orbitals could be $p_{\pi}$ (and maybe $d_{\pi}$ ) functions centered on each nitrogen atom, or they could be $d_{\pi}$ obitals centered at the midpoint of the $\mathrm{N}-\mathrm{N}$ bond. Either choice can be used because one only needs a basis capable of describing the large-r $L=2$ character of the metastable ${ }^{2} \Pi_{g}$ state's wave function. One usually would not add just one such function; rather several such functions, each with an orbital exponent $\alpha_{\text {j }}$ that characterizes its radial extent, would be used. Let us assume, for example, that $\mathrm{K}$ such additional diffuse $\pi$ functions have been used.

Next, using the conventional atomic orbital basis as well as the $K$ extra $\pi$ basis functions, one carries out an EOM calculation for the EA of the $\mathrm{N}_{2}$ molecule. In this calculation, one tabulates the energies of many (say M) of the EOM EA eigenvalues. One then scales the orbital exponents $\left\{\alpha_{j}\right\}$ of the K extra $\pi$ basis orbitals by a factor $\eta: \alpha_{j} \rightarrow \eta \alpha_{j}$ and repeats the calculation of the energies of the $M$ lowest EOM eigenvalues. This scaling causes the extra $\pi$ basis orbitals to contract radially (if $\eta>1$ ) or to expand radially (if $\eta<1$ ). It is this basis orbital expansion and contraction that produces expansion and contraction of the "box" discussed above. That is, one does not employ a box directly; instead, one varies the radial extent of the more diffuse basis orbitals to simulate the box variation.

If the conventional orbital basis is adequate, one finds that the extra $\pi$ orbitals, whose exponents are being scaled, do not affect appreciably the energy of the neutral $\mathrm{N}_{2}$ system. This can be probed by plotting the $\mathrm{N}_{2}$ energy (computed as $\langle 0, \mathrm{~N}|\mathrm{H}| 0, \mathrm{~N}\rangle$ ) as a function of the scaling parameter $\eta$; if the energy varies little with $\eta$, the conventional basis is adequate. 
In contrast to plots of the neutral $\mathrm{N}_{2}$ energy vs $\eta$, plots of the energies of the $\mathrm{M} \mathrm{N}_{2}{ }^{-1}$ anion states relative to the energy of $\mathrm{N}_{2}$, obtained as EOM eigenvalues, show significant $\eta$-dependence as Fig. 1 illustrates.

What does such a stabilization plot tell us and what do the various branches of the plot mean? First, we notice that each of the plots of the energy of an anion state (relative to the neutral molecule's energy, which is independent of $\eta$ ) grows with increasing $\eta$. This $\eta$-dependence arises from the $\eta$-scaling of the extra diffuse $\pi$ basis orbitals. Because most of the amplitude of such basis orbitals lies outside the valence region, the kinetic energy is the dominant contributor to such states' relative energies. Because $\eta$ enters into each orbital as $\exp \left(-\eta \alpha r^{2}\right)$, and because the kinetic energy operator involves the second derivative with respect to $r$, the kinetic energies of orbitals dominated by the diffuse $\pi$ basis functions vary as $\eta^{2}$. It is this quadratic growth with $\eta$ that appear as the basic trends in the energies vs $\eta$ plots in Fig. 1.

For small $\eta$, all of the $\pi$ diffuse basis functions have their amplitudes concentrated at large $r$ and have low kinetic energy. As $\eta$ grows, these functions become more radially compact and their kinetic energies grow just as the particle-in-a-box energies grow as the box length decreases. For example, note the three lowest energies shown above in Fig. 1 increasing from near zero as $\eta$ grows. As $\eta$ further increases, one reaches a point at which the third and fourth anion-state energies in Fig. 1 undergo an avoided crossing. At higher $\eta$ values, it is the second and third states and then the first and

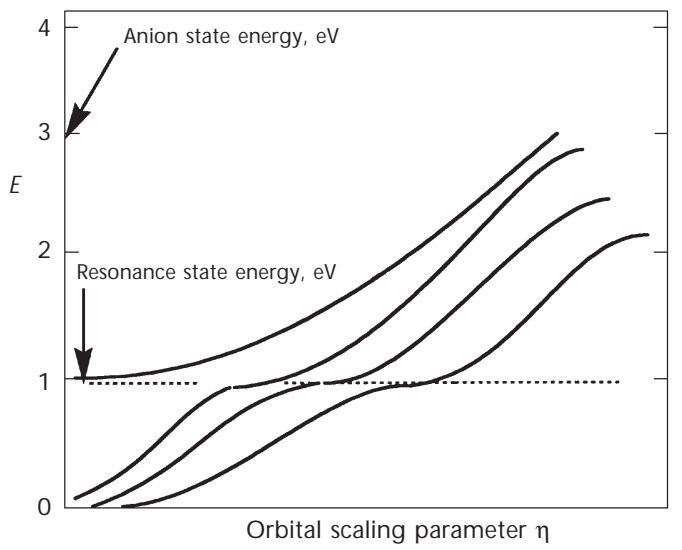

FIG. 1

Plots of the EOM EA eigenvalues for several anion states vs the orbital scaling parameter $\eta$. Note the avoided crossing of state energies near $1 \mathrm{eV}$ 
second states whose energies undergo such avoided crossings. At such $\eta$ values, if one examines the nature of the two anion wave functions (obtained as in Eq. (4)) whose energies avoid one another, one finds they contain substantial amounts of both valence and extra diffuse $\pi$ function character. Just to the left of the avoided crossing, the lower-energy state (the third state in Fig. 1 for the smallest $\eta$ at which an avoided crossing occurs) contains predominantly extra diffuse $\pi$ orbital character, while the higher-energy state (the fourth state) contains largely valence $\pi^{*}$ orbital character. To the right of the avoided crossing, the situation is reversed the lower-energy state (the third state in Fig. 1 for small $\eta$ ) contains predominantly valence orbital character, while the higher-energy state (the fourth state) contains largely diffuse orbital character.

However, at the special values of $\eta$ where the two states nearly cross, the kinetic energy of the diffuse state (as well as its radial size and local de Broglie wavelength) are appropriate to connect properly with the valence state to form a single resonance state. By connect properly we mean that the two states have wave function amplitudes, phases, and radial slopes that match. It is such boundary condition matching of valence-range and long-range character in the wave function that the stabilization method achieves. So, at such special $\eta$ values, one can achieve a description of the resonance state that correctly describes this state both in the valence region and in the large-r region. Only by tuning the energy of the large $r$ states using the $\eta$ scaling can one obtain this proper boundary condition matching.

Another observation helps to understand the content of such stabilization plots. One considers the density of states (i.e., how many states are there between energy $E$ and $E+d E$ for a fixed small value of $d E$ ?) in a plot such as Fig. 1. Clearly, in the range of energies near the avoided crossings, there is an enhanced density of states, while the state density is lower at "off-resonance" energies. When viewed either from the point of view of state densities or avoided crossings, there is something special about the region of energies near such resonances. As noted above, it is the fact that the valence range and continuum components of the wave function can be properly matched at such energies that is "special".

If one attempts to study metastable anion states without carrying out such a stabilization study, one is doomed to failure, even if one employs an extremely large and flexible set of diffuse basis functions. In such a calculation, one will certainly obtain a large number of anion "states" with energies lying above that of the neutral, but one will not be able to select from these states the one that is the true resonance state because the true state will be buried in the myriad of "states" representing the $\mathrm{N}_{2}+$ e continuum. 
In summary, by carrying out a series of anion-state energy calculations for several states and plotting them vs $\eta$, one obtains a stabilization graph. By examining this graph and looking for avoided crossings, one can identify the energies at which metastable resonances occur. It is absolutely critical to identify these resonance energies if one wishes to probe metastable anions. It is also possible $e^{28}$ to use the shapes (i.e., the magnitude of the energy splitting between the two states and the slopes of the two avoiding curves) of the avoided crossings in a stabilization graph to compute the lifetimes of the metastable states. Basically, the larger the avoided-crossing energy splitting between the two states, the shorter is the lifetime of the resonance state.

\section{CONCLUSIONS}

We have tried to illustrate how, by focusing on the intensive energies that one wishes to compute when studying EAs, IPS, or electronic excitation energies, one can replace the solution of the Schrödinger equation by the solution of so-called equations of motion. It is the eigenvalues of these EOMs that produce the EAs and IPs directly. We have reviewed some of the history of the development of EOM theory, especially as it applied to EAs and IPs, and we have attempted to show its relationships to Greens functions and extended Koopmans' theorem approaches to these same intensive energies. We have shown that a wide variety of EOM theories can be developed depending on how one chooses to describe the neutral molecule's wave function (i.e., in MP, MC-SCF, or CC fashion). Finally, we discussed some of the pitfalls that one encounters when applying EOM theory to EAs of molecules whose anion states are not bound but are metastable resonance states. It is our hope and belief that EOM methods have proven useful computationally and for gaining insight and will continue to have a bright future.

Readers who wish to learn more about how molecular EAs (and, to a lesser extent, IPs) have been studied theoretically are directed to this author's web site http://simons.hec.utah.edu as well as to a series ${ }^{29}$ of his reviews and chapters. The species that this group have examined include dipole-bound anions, zwitterion ions, conventional valence anions, multiply charged anions, as well as a wide variety of metastable anions.

This work was supported by NSF grants No. 9982420 and No. 0240387. 


\section{REFERENCES}

1. Rienstra-Kiracofe J. C., Tschumper G. S., Schaefer H. F., III, Nandi S., Ellison G. B.: Acc. Chem. Res. 2002, 102, 231.

2. Herzberg G.: Molecular Spectra and Molecular Structure I, p. 512. Van Nostrand, New York 1950.

3. a) Gutsev G. I., Boldyrev A. I.: Adv. Chem. Phys. 1985, 61, 169; b) Baker J., Nobes R. H., Radom L.: J. Comput. Chem. 1986, 7, 349; c) Simons J., Jordan K. D.: Chem. Rev. 1987, 87, 535; d) Kalcher J., Sax A. F.: Chem. Rev. 1994, 94, 2291; e) Kalcher J.: J. Ann. Rep., Sect. C, R. Soc. Chem. 1996, 93, 147; f) Berry R. S.: Chem. Rev. 1969, 69, 533.

4. See lit. ${ }^{3 \mathrm{f}}$ and references contained therein. Some of the earlier successes are given in the following: a) Clementi E., McLean A. D.: Phys. Rev. A 1964, 133, 419; b) Clementi E., McLean A. D., Raimondi D. L., Yoshimine M.: Phys. Rev. A 1964, 133, 1274; c) Clementi E.: Phys. Rev. A 1964, 135, 980; d) Pekeris C. L.: Phys. Rev. 1958, 112, 1649; e) Weiss A. W.: Phys. Rev. 1968, 166, 70; f) Cade P. E.: J. Chem. Phys. 1967, 47, 2390; g) Cade P. E.: Proc. R. Soc. A 1967, 91, 842; h) Taylor S., Harris F. E.: J. Chem. Phys. 1963, 39, 1012; i) Bondybey V., Pearson P. K., Schaefer H. F.: J. Chem. Phys. 1972, 57, 1123; j) Feller D., Davidson E. R.: J. Chem. Phys. 1989, 90, 1024; k) Feller D., Davidson E. R.: J. Chem. Phys. 1985, 82, 1024. In addition to lit. ${ }^{1}$, good sources for EA, IP data include: 1) Hotop H., Lineberger W. C.: J. Phys. Chem. Ref. Data 1975, 4, 539; m) Hotop H., Lineberger W. C.: J. Phys. Chem. Ref. Data 1985, 14, 731; n) Andersen T., Haugen H. K., Hotop H.: J. Phys. Chem. Ref. Data 1999, 28, 1511; o) Janousek B. K., Brauman J. I. in: Gas Phase Ion Chemistry (M. T. Bowers, Ed.), Vol. 2, p. 53. Academic Press, New York 1979; p) Miller T. M. in: CRC Handbook of Chemistry and Physics (R. C. West, M. J. Astle and W. H. Beyer, Eds), 74th ed., pp. 10-180-10-191. CRC Press, Boca Raton (FL) 1993; q) Bartmess J. E. in: NIST Chemistry Web-Book, NIST Standard Reference Database Number 69 (W. G. Ballard and P. J. Linstrom, Eds). National Institute of Standards, Technology, Gaithersburg (MD) February 2000. http://webbook.nist.gov; r) Franklin J. L., Dillard J. G., Field F. H.: Ionization Potentials, Appearance Potentials, and Heats of Formation of Gaseous Positive Ions. Natl. Stand. Ref. Data Ser. Natl. Bur. Stand. (U.S.) 26, 1969.

5. Much of what is presently known about atomic and molecular EAs can be found in lit. ${ }^{3,4}$ as well as in the following: a) Massey H.: Negative Ions. Cambridge University Press, Cambridge 1976; b) Branscomb L. M.: Atomic and Molecular Processes (D. R. Bates, Ed.). Academic Press, New York 1962.

6. Some of the early chemistry developments of Greens functions include: a) Goscinski O., Lukman B.: Chem. Phys. Lett. 1970, 6, 573; b) Jørgensen P., Oddershede J.: J. Chem. Phys. 1972, 57, 277; c) Linderberg J., Jørgensen P., Oddershede J., Ratner M.: J. Chem. Phys. 1972, 56, 6213; d) Csanak G., Taylor H. S., Yaris R.: Phys. Rev. A: At., Mol., Opt. Phys. 1971, 3, 6213; e) Csanak G., Taylor H. S., Yaris R.: Adv. At. Mol. Phys. 1971, 7, 288; f) Linderberg J., Öhrn Y.: J. Chem. Phys. 1968, 49, 716; g) Linderberg J., Öhrn Y.: Chem. Phys. Lett. 1967, 1, 295; h) Linderberg J., Öhrn Y.: Proc. R. Soc., Sect. A 1963, 185, 445; i) Öhrn Y., Linderberg J.: Phys. Rev. A 1965, 139, 1063; j) Linderberg J., Ratner M.: Chem. Phys. Lett. 1970, 6, 37; k) Linderberg J., Thulstrup E. W.: J. Chem. Phys. 1968, 49, 710; 1) Reinhardt W. P., Doll J. D.: J. Chem. Phys. 1969, 50, 2767; m) Schneider B., Taylor H. S., Yaris R.: Phys. Rev. A: At., Mol., Opt. Phys. 1970, 1, 855; n) Doll J. D., Reinhardt W. P.: J. Chem. Phys. 1972, 57, 1169; o) Jørgensen P., Simons J.: J. Chem. Phys. 1975, 63, 5302; p) Linderberg J., Öhrn Y.: Propagators in Quantum Chemistry. Academic Press, London 
1973; q) Cederbaum L. S., Holneicher G., Peyerimhoff S.: Chem. Phys. Lett. 1971, 11, 421; r) Cederbaum L. S., Holneicher G., von Niessen W.: Chem. Phys. Lett. 1973, 18, 503; s) Cederbaum L. S.: Theor. Chim. Acta 1973, 31, 239; t) Yarlagadda B. S., Csanak Gy., Taylor H. S., Schneider B., Yaris R.: Phys. Rev. A: At., Mol., Opt. Phys. 1973, 7, 146; u) Pickup B. T., Goscinski O.: Mol. Phys. 1973, 36, 1013; v) Purvis G. D., Öhrn Y.: J. Chem. Phys. 1974, 60, 4063; w) Purvis G. D., Öhrn Y.: J. Chem. Phys. 1975, 62, 2045; x) For a more recent overview, see Ortiz J. V., Leszczynski J. (Eds): Computational Chemistry: Reviews of Current Trends, Vol. 2, p. 1. World Scientific, Singapore 1997; y) Purvis G. D., Öhrn Y.: Int. J. Quantum Chem., Quantum Chem. Symp. 1977, 11, 359.

7. A good overview of time-dependent response function theory, including linear and non-linear response functions is offered in Olsen J., Jorgensen P. in: Modern Electronic Structure Theory (D. Yarkony, Ed.), p. 857. World Scientific, Singapore 1995.

8. a) Dunning T. H., McKoy V.: J. Chem. Phys. 1967, 47, 1735; b) Dunning T. H., McKoy V.: J. Chem. Phys. 1968, 48, 5263; c) Shibuya T. I., McKoy V.: J. Chem. Phys. 1970, 53, 2208.

9. a) Rowe D. J.: Rev. Mod. Phys. 1968, 40, 153; b) Rowe D. J.: Nuclear Collective Motion Models and Theory. Methuen, London 1970.

10. a) Simons J., Smith W. D.: J. Chem. Phys. 1973, 58, 4899. This work exploited developments made earlier in studies of excited electronic states and reduced density matrices: b) Simons J.: J. Chem. Phys. 1971, 55, 1218; c) Simons J.: J. Chem. Phys. 1972, 57, 3787.

11. a) Simons J., Jørgensen P.: J. Chem. Phys. 1976, 64, 1413; b) Tsung-Tai Chen, Simons J., Jordan K. D.: Chem. Phys. 1976, 14, 145; c) Simons J.: J. Chem. Phys. 1976, 64, 4541; d) Simons J.: Int. J. Quantum Chem. 1977, 12, 227; e) Dalgaard E., Simons J.: J. Phys. B: At., Mol. Opt. Phys. 1977, 10, 2767; f) Simons J.: Annu. Rev. Phys. Chem. 1977, 28, 15; g) Banerjee A., Shepard R., Simons J.: Int. J. Quantum Chem., Quantum Chem. Symp. 1978, 12, 389; h) Donnelly R. A., Simons J.: J. Chem. Phys. 1980, 73, 2858.

12. See, for example: a) Jørgensen P., Simons J.: Second Quantization Based Methods in Quantum Chemistry. Academic Press, New York 1981; b) Helgaker T., Jørgensen P., Olsen J.: Modern Electronic Structure Theory. J. Wiley, New York 2000.

13. Manne R.: Chem. Phys. Lett. 1977, 45, 470.

14. The first- and second-order density matrices, respectively, have elements given by $\langle 0, \mathrm{Nl}$ $j^{+} k|0, \mathrm{~N}\rangle$ and $\left\langle 0, \mathrm{Nl} j^{+} k^{+} 1 h \mid 0, \mathrm{~N}\right\rangle$.

15. a) Simons J., Chen T.-T., Smith W. D.: Chem. Phys. Lett. 1974, 26, 296; b) Smith W. D., Chen T.-T., Simons J.: Chem. Phys. Lett. 1974, 27, 499; c) Griffing K. M., Simons J.: J. Chem. Phys. 1975, 62, 535; d) Kenney J., Simons J.: J. Chem. Phys. 1975, 62, 592; e) Griffing K., Kenney J., Simons J., Jordan K.: J. Chem. Phys. 1975, 63, 4073; f) Griffing K., Simons J.: J. Chem. Phys. 1976, 64, 3610; g) Jordan K. D., Griffing K. M., Kenney J., Andersen E. L., Simons J.: J. Chem. Phys. 1976, 64, 4730; h) Andersen E., Simons J.: J. Chem. Phys. 1976, 64, 4548; i) Jordan K. D., Simons J.: J. Chem. Phys. 1976, 65, 1601; j) Andersen E., Simons J.: J. Chem. Phys. 1976, 65, 5393; k) Andersen E., Simons J.: J. Chem. Phys. 1977, 66, 2427.

16. Feyereisen M., Nichols J., Oddershede J., Simons J.: J. Chem. Phys. 1992, 96, 2978.

17. See, for example: Ortiz J. V., Leszczynski J. (Eds): Computational Chemistry: Reviews of Current Trends, Vol. 2, p. 1. World Scientific, Singapore 1997.

18. a) Frisch M. J., Trucks G. W., Schlegel H. B., Scuseria G. E., Robb M. A., Cheeseman J. R., Montgomery J. A., Jr., Vreven T., Kudin K. N., Burant J. C., Millam J. M., Iyengar S. S., Tomasi J., Barone V., Mennucci B., Cossi M., Scalmani G., Rega N., Petersson G. A., H. 
Nakatsuji, Hada M., Ehara M., Toyota K., Fukuda R., Hasegawa J., Ishida M., Nakajima T., Honda Y., Kitao O., Nakai H., Klene M., Li X., Knox J. E., Hratchian H. P., Cross J. B., Adamo C., Jaramillo J., Gomperts R., Stratmann R. E., Yazyev O., Austin A. J., Cammi R., Pomelli C., Ochterski J. W., Ayala P. Y., Morokuma K., Voth G. A., Salvador P., Dannenberg J. J., Zakrzewski V. G., Dapprich S., Daniels A. D., Strain M. C., Farkas O., Malick D. K., Rabuck A. D., Raghavachari K., Foresman J. B., Ortiz J. V., Cui Q., Baboul A. G., Clifford S., Cioslowski J., Stefanov B. B., Liu G., Liashenko A., Piskorz P., Komaromi I., Martin R. L., Fox D. J., Keith T., Al-Laham M. A., Peng C. Y., Nanayakkara A., Challacombe M., Gill P. M. W., Johnson B., Chen W., Wong M. W., Gonzalez C., Pople J. A.: Gaussian, Inc., Pittsburgh (PA) 2003; b) Ortiz J. V.: Adv. Quantum Chem. 1999, 35, 33; c) Propagating insight: A tribute to the works of Öhrn Y., Ortiz J. V., Kurtz H. A. (Eds): Adv. Quantum Chem. 35, Academic Press, New York 1999.

19. Morrel M. M., Parr R. G., Levy M.: J. Chem. Phys. 1975, 62, 549.

20. a) Day O. W., Smith D. W., Garrod C.: Int. J. Quantum Chem., Quantum Chem. Symp. 1974, 8, 501; b) Smith D. W., Day O. W.: J. Chem. Phys. 1975, 62, 113.

21. Andersen E., Simons J.: J. Chem. Phys. 1977, 66, 1067.

22. Cioslowski J., Piskorz P., Liu G.: J. Chem. Phys. 1997, 107, 6804.

23. Katriel J., Davidson E. R.: Proc. Natl. Acad. Sci. 1980, 77, 4403.

24. a) Paldus J.: Proceedings of the Fourth International Congress of Quantum Chemistry, p. 31. D. Reidel, Dordrecht 1983; b) Č́ižek J., Paldus J.: Int. J. Quantum Chem. 1971, 5, 359.

25. Among the first workers to develop the requisite EOM machinery for a CC reference wave function were the following: a) Monkhorst H. J.: Int. J. Quantum Chem., Quantum Chem. Symp. 1977, 11, 421; b) The framework for applying EOM-CC theory to EAs was developed in: Nooijen M., Bartlett R. J.: J. Chem. Phys. 1995, 102, 3629. The use of CC wave functions and EOM-type theories to compute excitation energies was advanced by several workers including the following: c) Mukhopadhyay D., Mukhopadhyay S., Chaudhuri R., Mukherjee D.: Theor. Chim. Acta 1991, 80, 441; d) Stanton J. F., Bartlett R. J.: J. Chem. Phys. 1993, 98, 7029; e) Piecuch P., Kowalski K. in: Computational Chemistry: Reviews of Current Trends (J. Leszczynski, Ed.), Vol. 5, p. 1. World Scientific, Singapore 2000. A special focus on implementing CC-based theories for electron affinities and ionization potentials was made by Nooijen in the following: f) Nooijen M.: Ph.D. Thesis. Vrije Universiteit Amsterdam, Amsterdam 1992; g) Nooijen M., Bartlett R. J.: J. Chem. Phys. 1997, 106, 6449; h) Stanton J. F., Gauss J.: J. Chem. Phys. 1994, 101, 8938.

26. A good overview is given in Bartlett R. J., Stanton J. F. in: Reviews in Computational Chemistry (K. B. Lipkowitz and D. B. Boyd, Eds), Vol. 5. VCH, New York 1994.

27. Hazi A. U., Taylor H. S.: Phys. Rev. A: At., Mol., Opt. Phys. 1970, 1, 1109.

28. a) Simons J.: J. Chem. Phys. 1981, 75, 2465; b) Frey R. F., Simons J.: J. Chem. Phys. 1986, 84,4462 .

29. a) Simons J., Skurski P.: Recent Res. Devel. Phys. Chem., Theoretical Prospect of Negative Ions (J. Kalcher, Ed.), 2002, 117; b) Encyclopedia of mass spectrometry, Vol. 5. Theory and Ion Chemistry 2. Theory (Energies and Potential Energy Surfaces). Anions, Simons J., 2002, 55; c) Gutowski M., Skurski P., Jordan K. D., Simons J.: Int. J. Quantum Chem. 1997, 64, 183; d) Simons J.: Int. J. Quantum Chem., Quantum Chem. Symp. 1982, 16, 575; e) Simons J.: Theoretical Chemistry: Advances and Perspectives, Vol. 3. Academic Press, New York 1978. 